\title{
Modeling, Analysis, and Experimental Validation of Frequency Dividers with Direct Injection
}

\author{
Antonio Buonomo and Alessandro Lo Schiavo \\ Facoltà di Ingegneria, Seconda Università degli Studi di Napoli, Via Roma, 29-81031 Aversa CE, Italy \\ Correspondence should be addressed to Antonio Buonomo; antonio.buonomo@unina2.it
}

Received 30 December 2012; Accepted 8 March 2013

Academic Editor: Jenny Liu

Copyright (C) 2013 A. Buonomo and A. Lo Schiavo. This is an open access article distributed under the Creative Commons Attribution License, which permits unrestricted use, distribution, and reproduction in any medium, provided the original work is properly cited.

\begin{abstract}
An analytical model and a methodology are presented for the analysis of CMOS injection-locked frequency dividers with direct injection, which are used in modern wireless communication systems. The amplitude and phase of the oscillation in the synchronous operation mode, as well as the locking range, are found in explicit form. The width of the locking range is determined by the condition of the existence and stability of synchronous oscillations. The accuracy of the model and of the presented formulas is validated through a comparison with experimental results, which are in good agreement with the analytical ones.
\end{abstract}

\section{Introduction}

The possibility offered by the modern RF-CMOS technology to realize on-chip injection-locked frequency dividers (ILFDs) has led to a renewed interest for the study of the behavior of LC oscillators under the action of an external signal, firstly performed in a pragmatic, and effective, manner in a pioneering paper by Adler [1] and then in $[2,3]$. Many attempts have been devoted to seeking topologies able to broaden the locking range [4] and to increase the division ratio. A number of topologies of ILFDs have been devised to perform the frequency division with a power consumption lower than that of digital dividers and thus usable advantageously in PLLs.

Investigations were also devoted to the issue of developing a model of the ILFDs and a methodology for their analysis, at first addressed in [5-7] and subsequently in many papers (see [8] and references therein), which is motivated by the need for a better understanding of their operation in order to provide useful design insights. Among these, mention should be made of papers $[9,10]$ where a comprehensive methodology is provided for the analysis of most commonly used topologies of ILFDs, namely, with injection through a tail device and with direct injection.

However, the frequency dividers with direct injection have not been sufficiently treated in the literature from an analytical point of view, although they are widely used in applications for a very low input capacitance [11, 12] and a wide locking range $[11,13]$, which is one of the most important figures of merit of ILFDs. In the present work, we wish to demonstrate the accuracy of the model of ILFDs developed in [9], here made more general, as well as the accuracy of the methodology for their analysis under steady-state operating conditions. This allows us to determine the amplitude and the phase shift of the locked oscillation, as well as the locking range by analytical expressions. While the methodology was tested in previous papers through numerical simulations only, here the accuracy is verified for the first time through experimental tests on a circuit prototype that shows a good agreement between theory and experiments.

\section{Modeling}

A divide-by-2 injection-locked frequency divider with complementary topology and direct injection (Figure 1) can be modeled by the equivalent circuit in Figure 2 [9]. The model is formed by the parallel connection of an $L C$ tank, whose losses are represented by an equivalent resistance $R$, a nonlinear two-terminal transconductor described by the current-voltage relationship $i_{\mathrm{nl}}(v)$, and a controlled current source described by the current-voltage relationship 


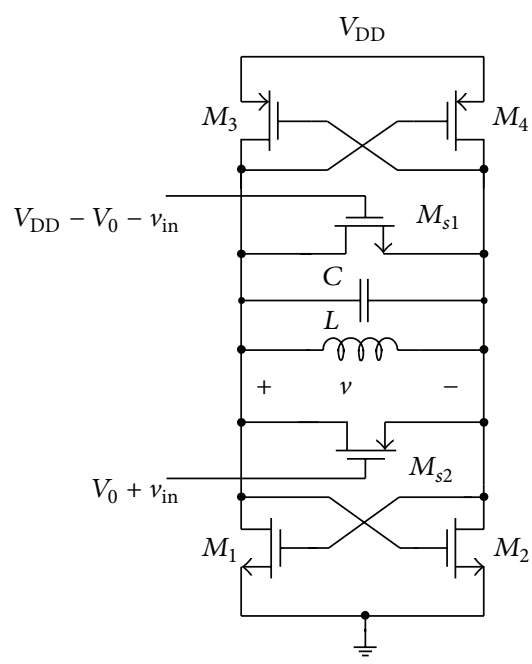

FIGURE 1: Circuit diagram of an injection-locked frequency divider with complementary topology and direct injection.

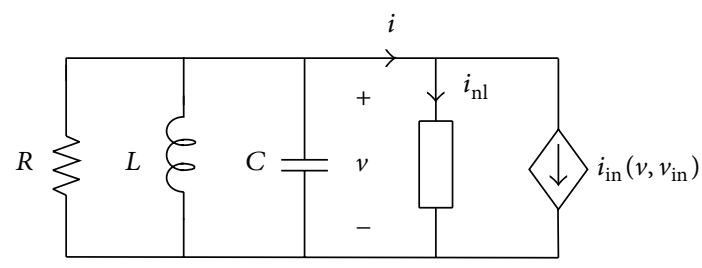

FIgURE 2: Equivalent model of the ILFD in Figure 1.

$i_{\text {in }}\left(v, v_{\text {in }}\right)$, where $v$ denotes the differential voltage between the drain nodes of the ILFD. The $L C$ tank and the transconductor represent the basic oscillator that is perturbed by the injection current $i_{\text {in }}\left(v, v_{\text {in }}\right)$, which depends on both the injection signal $v_{\text {in }}$, and the tank voltage $v$.

In the circuit in Figure 1, the complementary pairs of cross-coupled devices $\left(M_{1}-M_{2}, M_{3}-M_{4}\right)$ are sized to have complementary characteristics that set the bias voltages at the drain nodes to half the supply voltage, in order to maximize the oscillation swing. These devices form the composite two-terminal shown in Figure 3(a), which is described by a nonlinear characteristic, $i_{\mathrm{nl}}(v)$ [14-17]. It is easy to realize that $i_{\mathrm{nl}}(0)=0$ and, due to the circuit symmetry, $i_{\mathrm{nl}}(v)$ is an odd function of $v$. Since the two-terminal sub-circuit in Figure 3(a) has to compensate the tank losses, it exhibits a negative resistance, and thus, it is locally active with a currentvoltage characteristic $i_{\text {nl }}(v)$ lying into the second and fourth quadrants of the $i-v$ plane (Figure 5).

The two complementary MOS switches $\left(M_{s 1}, M_{s 2}\right)$ of the circuit in Figure 1 form the injection circuit shown in Figure $3(\mathrm{~b})$, which is driven by the external periodic signal $v_{\text {in }}$. Under the action of that signal, the injection circuit injects into the tank a current $i_{\text {in }}$ that depends in a nonlinear way on both the injection signal $v_{\text {in }}$ and the tank voltage $v$. Consequently, the injection circuit can be represented by a two-terminal element providing a controlled current described by the nonlinear function $i_{\text {in }}\left(v, v_{\text {in }}\right)$. Since the injection circuit perturbs the basic $L C$ oscillator in a passive way, $i_{\text {in }}\left(v, v_{\text {in }}\right)$ is depicted by a family of current-voltage curves lying into the first and third quadrants of the $i-v$ plane (Figure 6).

From the previous observations, it follows that the behavior of the ILFD in Figure 1 can be predicted through the equivalent circuit shown in Figure 2. The development of a behavioral model which allows us to analyze the divider in Figure 1 by an analytical approach relies on the possibility of identifying the functions $i_{\text {nl }}(v)$ and $i_{\text {in }}\left(v, v_{\text {in }}\right)$ through few parameters, which is possible, as we propose to show.

First, we observe that usually the dividers operate with small amplitudes of the injection signal, that is, with $v_{\text {in }}$ small enough to make reasonable the following approximation:

$$
i_{\text {in }}\left(v, v_{\text {in }}\right) \approx i_{\text {in }}(v, 0)+g(v) v_{\text {in }},
$$

where

$$
g(v)=\left.\frac{\partial i_{\mathrm{in}}}{\partial v_{\mathrm{in}}}\right|_{v_{\mathrm{in}}=0} \approx \frac{i_{\mathrm{in}}\left(v, V_{\mathrm{inDC}}\right)-i_{\mathrm{in}}\left(v,-V_{\mathrm{inDC}}\right)}{2 V_{\mathrm{inDC}}},
$$

$V_{\text {inDC }}$ being a constant voltage applied to the inputs, in addition to the bias voltages $V_{0}$ and $V_{\mathrm{DD}}-V_{0}$. Then, we observe that nonlinear functions $i_{\text {nl }}(v), i_{\text {in }}(v, 0)$, and $g(v)$ can be easily measured at the circuit terminals in Figures 3(a) and 3(b). To show this, a prototype of the circuit in Figure 1 was implemented using integrated circuits ST HCF4007UB (dual complementary pairs plus inverter) powered by a $V_{\mathrm{DD}}=5 \mathrm{~V}$ battery. While NMOS and PMOS in Figure 3(b) were implemented using a single device of the integrated circuit, each of the four cross-coupled active devices in Figure 3(a) was implemented with the parallel connection of two transistors of the integrated circuit to increase the transconductance parameter.

In order to measure the nonlinear characteristics of the circuit under test, that is, $i_{\mathrm{nl}}(v), i_{\mathrm{in}}(v, 0)$, and $g(v)$, the auxiliary circuit in Figure 4 was connected to the nodes $x$ and $y$ of the circuit in Figure 3(a) and, then, of the circuit in Figure 3(b). Voltages at these nodes, formed by a bias component equal to $V_{\mathrm{DD}} / 2$ and an $\mathrm{AC}$ component, were applied through $100 \Omega$ series resistors that allow us to measure the absorbed currents using voltage buffers and opamp differential amplifiers. While in case of perfect matching of the transistors in Figure 3(a) we measure $i_{1}=i_{2}$, in case of transistor mismatch we measure slightly different currents. Therefore, we calculate the mean value of these currents; that is, $i_{\mathrm{nl}}=\left(i_{1}+i_{2}\right) / 2$ for the circuit in Figure 3(a) and $i_{\text {in }}=$ $\left(i_{1}+i_{2}\right) / 2$ for the circuit in Figure $3(\mathrm{~b})$. If the circuit under test is the locally active two-terminal sub-circuit in Figure 3(a), the measured current $i_{\mathrm{nl}}$ is a function of the applied voltage $v$, as shown in Figure 5. The same figure shows that the cubic approximant [18-20]

$$
i_{\mathrm{nl}}(v)=a_{\mathrm{nl}} v+c_{\mathrm{nl}} v^{3}
$$

is in good agreement with the actual characteristic.

On the other hand, if the circuit under test is the injection circuit shown in Figure 3(b), the measured current is $i_{\text {in }}$ and it is plotted in Figure 6(a) as a function of the applied differential voltage, $v$, for different values of the applied input 


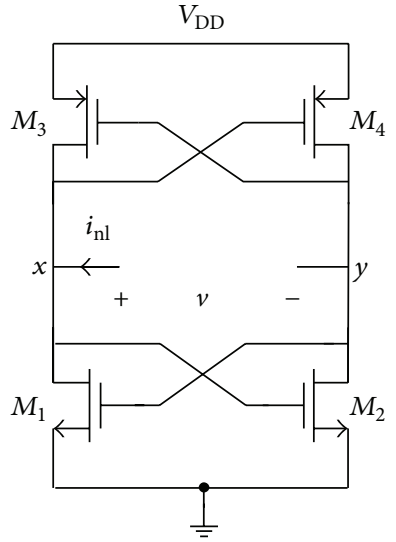

(a)

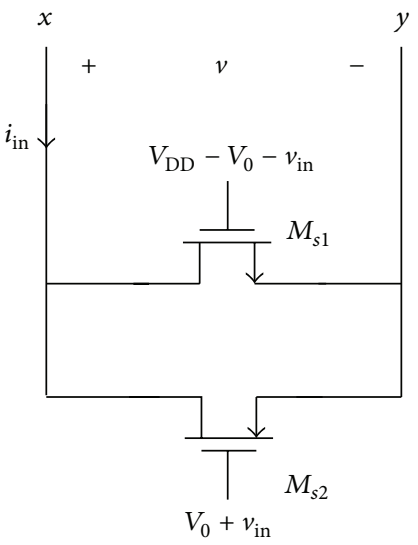

(b)

FIGURE 3: (a) Locally active two-terminal sub-circuit of the ILFD in Figure 1; (b) injection circuit of the ILFD in Figure $1 . V_{0}$ and $V_{\mathrm{DD}}-V_{0}$ are bias voltages.
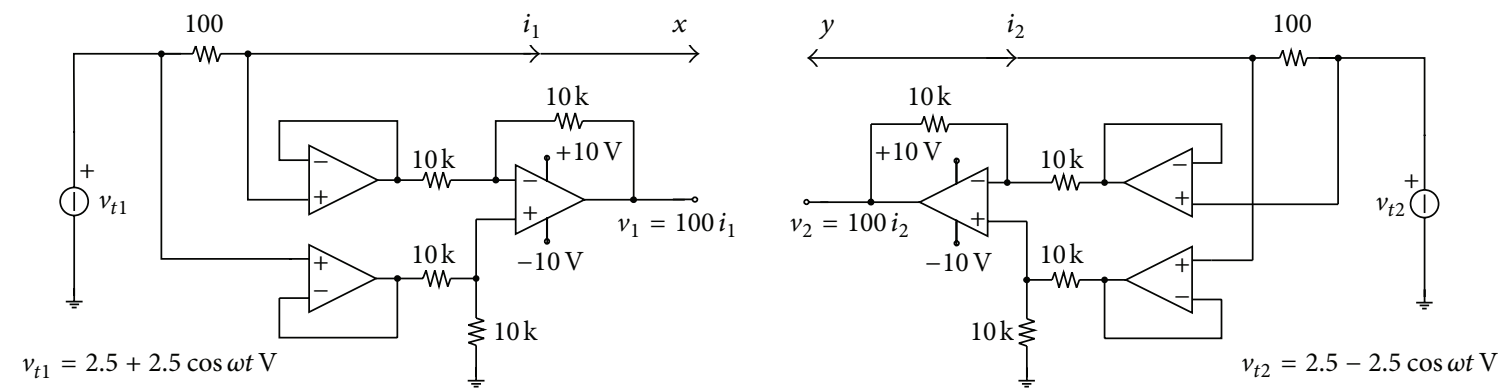

FIgURE 4: Auxiliary circuit used to apply voltages $v_{t 1}$ and $v_{t 2}$ to the circuits in Figures 3(a) and 3(b) and to measure the absorbed currents $i_{1}$ and $i_{2}$.

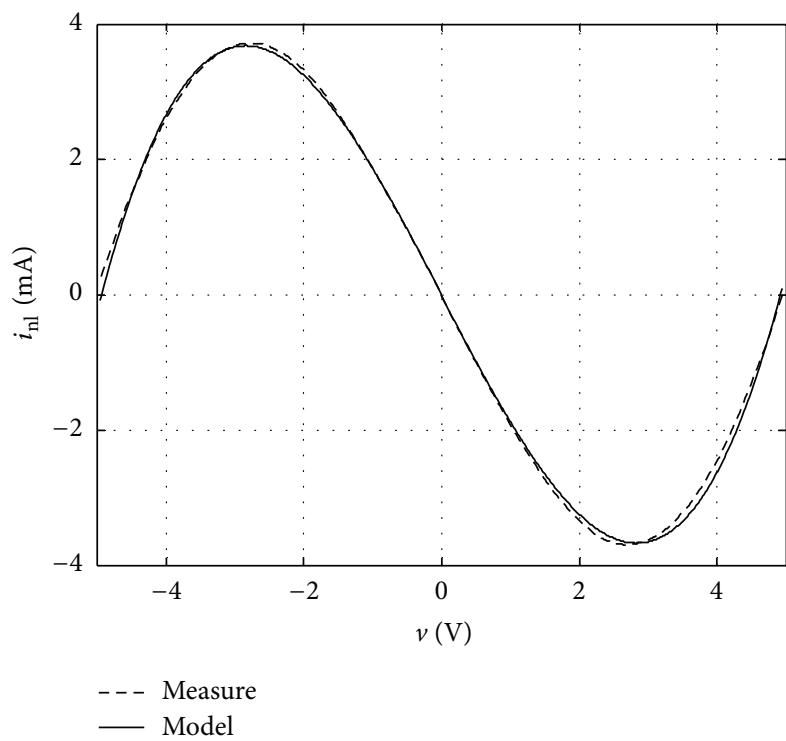

FIGURE 5: Nonlinear characteristic of the locally active two-terminal sub-circuit, shown in Figure 3(a) with $V_{\mathrm{DD}}=5 \mathrm{~V}$. Measurements are obtained using integrated circuits ST HCF4007UB and the testing circuit in Figure 4. Approximate model is obtained using a cubic polynomial with $a_{\mathrm{nl}}=-1.94 \mathrm{~mA} / \mathrm{V}$ and $c_{\mathrm{nl}}=80 \mathrm{uA} / \mathrm{V}^{3}$. voltage $v_{\text {in }}=V_{\text {inDC. }}$. Figure $6(\mathrm{a})$ shows that $i_{\text {in }}\left(v, v_{\text {in }}=0\right)$ can be modeled by a cubic polynomial, including a quadratic odd function $b_{\text {in }} v|v|$; that is, $i_{\text {in }}(v, 0)=a_{\text {in }} v+b_{\text {in }} v|v|+c_{\text {in }} v^{3}$, where $|v|$ denotes the absolute value of $v$. As shown in Figure 6(a), the approximated function fits well the actual characteristic. Exploiting the measured curves $i_{\text {in }}(v, 1)$ and $i_{\text {in }}(v,-1)$, shown in Figure 6(a), it is possible to estimate the function $g(v)$ according to (2) as shown in Figure 6(b). Even if a fifth-order polynomial provides a very good approximation for $g(v)$, a linear approximation; that is, $g(v) \approx k v$, is sufficient to obtain simple and quite accurate analytical results, as we show in Section 4 . Thus, the injection nonlinearity takes the following expression:

$$
i_{\text {in }}\left(v, v_{\text {in }}\right)=a_{\text {in }} v+b_{\text {in }} v|v|+c_{\text {in }} v^{3}+k v v_{\text {in }} .
$$

The schematization of the divider as in Figure 2 and the identification of the functions (3) and (4) allow us to describe the circuit operation with the following model

$$
\left(D^{2}+\frac{\omega_{0}}{Q} D+\omega_{0}^{2}\right) v=-D \frac{\omega_{0} R}{Q}\left(i_{\mathrm{nl}}(v)+i_{\text {in }}\left(v, v_{\text {in }}\right)\right)
$$

obtained by the Kirchhoff's current law at the common node in Figure 2 and by using $D$ to denote the derivative operator 


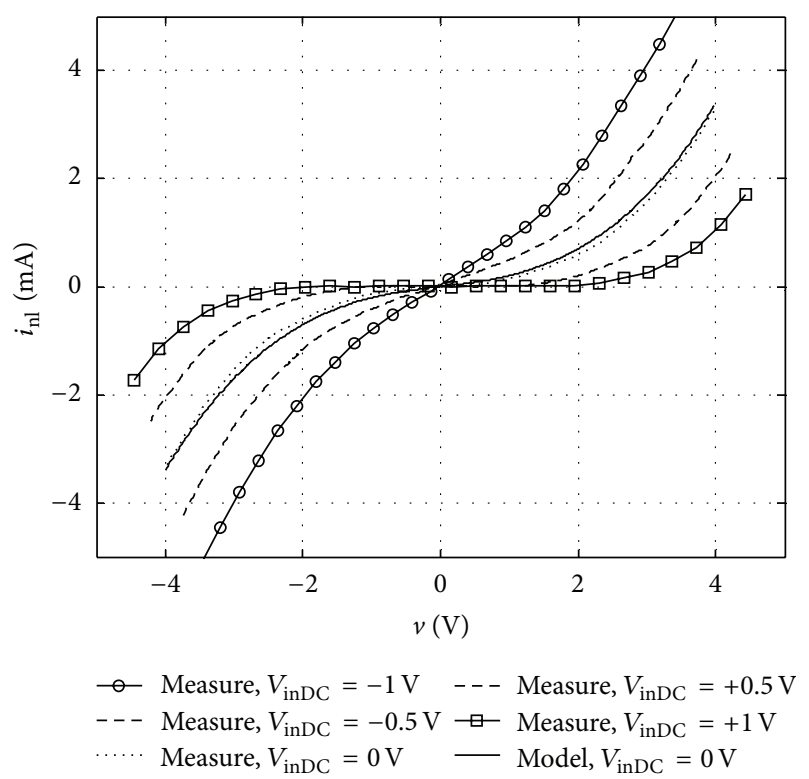

(a)

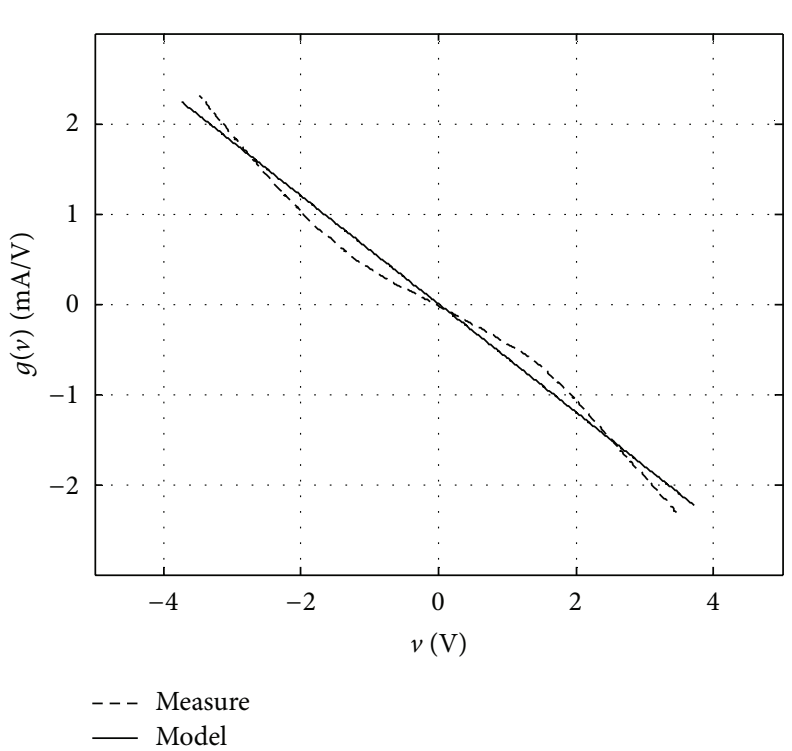

(b)

FIGURE 6: (a) Nonlinear characteristics of the injection circuit shown in Figure 3(b) for different values of the constant injection voltage $v_{\text {in }}=V_{\text {inDC }}$, obtained for $V_{\mathrm{DD}}=5 \mathrm{~V}$ and $V_{0}=1 \mathrm{~V}$. Measurements are obtained using integrated circuits ST HCF4007UB and the testing circuit in Figure 4. Approximate model for $v_{\text {in }}=0$ is obtained using cubic polynomial (4) with $a_{\text {in }}=0.13 \mathrm{~mA} / \mathrm{V}, b_{\text {in }}=40 \mathrm{uA} / \mathrm{V}^{2}$, and $c_{\text {in }}=35 \mathrm{uA} / \mathrm{V}^{3}$. (b) Voltage-dependent transconductance $g(v)$. Measurement's curve is obtained using (2) and curves shown in (a). Approximate model is obtained using the last term in (4) with $k=-0.6 \mathrm{~mA} / \mathrm{V}^{2}$.

with respect to time $t . \omega_{0}=1 / \sqrt{L C}$ is the resonant frequency of the tank and $Q=\omega_{0} \mathrm{RC}$ its quality factor.

It should be observed that the values assumed by the parameters of the algebraic characteristics (3) and (4) and used to model the transconductor and the injection circuit, obviously depend on the technology used as well as on the transistor sizes, but this modeling approach can be advantageously applied independently on the device technology. The parasitics and the delay of devices, due to the high frequency, are included, as a rule $[5,6,8]$, into the capacitance of the $L C$ tank. This simplifies the analysis, making it possible to capture the essential aspects of the synchronization phenomenon in ILFDs.

\section{Analysis}

If a sinusoidal synchronization signal $v_{\text {in }}=V_{\text {in }} \cos (2 \omega t)$ with frequency $\omega$ close to the tank resonant frequency $\omega_{0}=$ $1 / \sqrt{L C}$ is applied to the circuit in Figure 1, it operates as a divider-by-2 because, by virtue of the last term in (4), the injection circuit essentially performs a mixing between the input signal $v_{\text {in }}=V_{\text {in }} \cos (2 \omega t)$ and the tank voltage $v$. Consequently, the current injected into the tank has a component at fundamental frequency $\omega$, which determines the tank voltage, while the harmonics are filtered out by the $L C$ tank. We will therefore seek a solution to (5) in the form of a quasiharmonic function of time, namely, $v=$ $V(t) \cos [\omega t+\theta(t)]$. Here, $V(t)$ and $\theta(t)$ denote the envelope of the oscillation and the phase difference between the forced oscillation and the forcing signal, respectively, which do not vary in time when synchronization occurs. Thus, in order to reveal the locking conditions, we need to formulate the explicit equations describing the evolution of $V(t)$ and $\theta(t)$. Locking mean that there are stable fixed points in these equations, so we have to find the conditions for these points to exist and to be stable.

It should be observed that the exact periodic solution of (5) for arbitrary values of the parameters $\omega_{0}, Q, V_{\text {in }}$, and $\omega$ cannot be found analytically with the mathematical tools available. However, we can analytically find the approximate solutions that describe the unknown solutions with a certain degree of accuracy. This is possible resorting to the methods of asymptotic analysis for weakly nonlinear systems. Applying the well-known solution method of slowly varying amplitude and phase (SVA) to (5), as shown in detail in [9], we obtain a couple of equations for the amplitude and the phase, that is, the so-called averaging equations

$$
\begin{gathered}
\dot{V}(t)=-\frac{\omega_{0} a R}{2 Q} V\left(\frac{1}{a R}-1+\frac{8 b}{3 \pi} V+\frac{3}{4} c V^{2}-\frac{k_{c} V_{\text {in }}}{2} \cos 2 \theta\right), \\
\dot{\theta}(t)=\Delta-\frac{\omega_{0}}{Q} a R \frac{k_{c} V_{\text {in }}}{4} \sin 2 \theta,
\end{gathered}
$$

where the dot denotes the derivative with respect to $t, \Delta=$ $\omega_{0}-\omega$ is the frequency detuning of the output voltage $v, a=$ $-\left(a_{\mathrm{nl}}+a_{\mathrm{in}}\right), b=b_{\mathrm{in}} / a, c=\left(c_{\mathrm{nl}}+c_{\mathrm{in}}\right) / a$, and $k_{c}=-k / a$.

The formulation (6) of the described equations of the ILFD in terms of amplitude and phase is a useful tool for analysis. These equations have a fundamental importance in the theory of synchronization and their significance is due to 


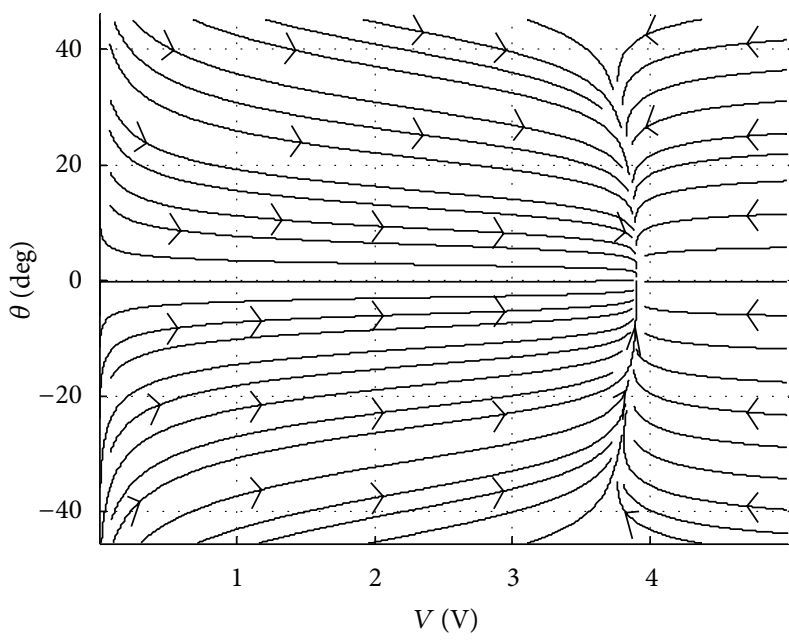

(a)

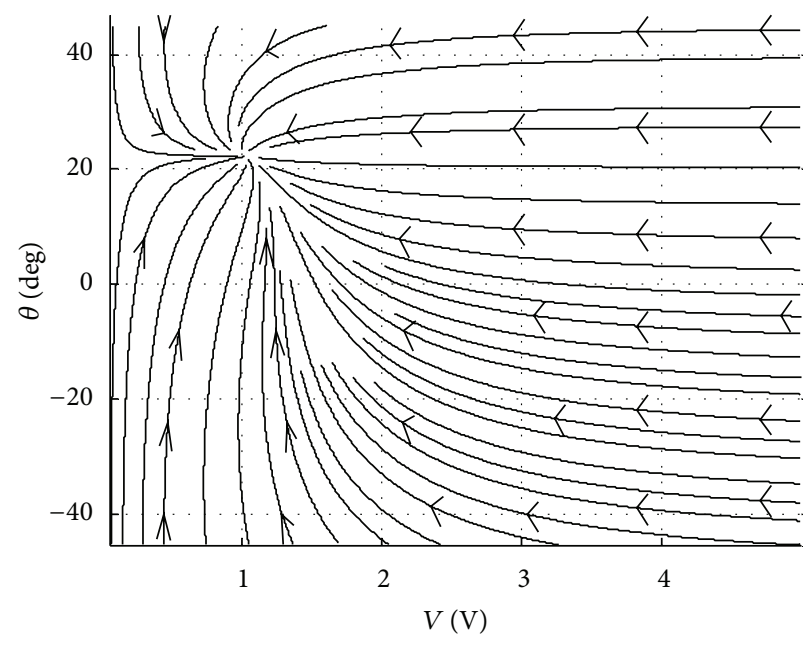

(b)

FIGURE 7: Phase portrait of system (6) with $b_{\text {in }}=40 \mu \mathrm{A} / \mathrm{V}^{2}, c_{\mathrm{nl}}+c_{\text {in }}=115 \mu \mathrm{A} / \mathrm{V}^{3}, k=-0.6 \mathrm{~mA} / \mathrm{V}^{2}, R=1950 \Omega$, and $V_{\text {in }}=0.5 \mathrm{~V}$. (a) for $a_{\mathrm{nl}}+a_{\mathrm{in}}=-1.81 \mathrm{~mA} / \mathrm{V}$ and $\Delta_{n}=0,(V, \theta)=\left(3.9 \mathrm{~V}, 0^{\circ}\right)$ is a stable node with $\lambda_{a}=-0.083$ and $\lambda_{b}=-0.763$; (b) for $a_{\mathrm{nl}}+a_{\mathrm{in}}=0.53 \mathrm{~mA} / \mathrm{V}$ and $\Delta_{n}=0.1,(V, \theta)=\left(1.02 \mathrm{~V}, 22.2^{\circ}\right)$ is a stable node with $\lambda_{a}=\lambda_{b}=-0.202$.

the fact that the analysis of (5) that is non autonomous, that is, depending explicitly on time, is reduced to the analysis of the autonomous system of (6). In terms of bifurcation theory, instead of analyzing the periodic orbits of (5), we can analyze the fixed points in (6), which is obviously much easier. This makes it possible to determine the following: (i) the amplitude and the phase of the output voltage in steady state, (ii) the locking range; (iii) the stability characteristics of locked oscillations, (iv) the amplitude and the phase of the output voltage during the transient preceding the steadystate. Note that previous approaches for analyzing ILFDs are limited only to the calculation of the locking range with the simplifying assumption that the amplitude of the output voltage is not dependent on the frequency $[1,5,6]$. The fixed points of (6) that correspond to $\dot{V}=\dot{\theta}=0$ can be found by solving the resulting nonlinear algebraic system for the amplitude $V$ and the phase $\theta$. It can be shown that this system can be solved in a closed form [9], leading to the following physically meaningful solution:

$$
\begin{gathered}
V=\sqrt{\frac{4}{3 c} \frac{a R-1}{a R}+\frac{2 k_{c} V_{\mathrm{in}}}{3 c} \sqrt{1-\left(\frac{4 C \Delta}{a k_{c} V_{\mathrm{in}}}\right)^{2}}+\left(\frac{16 b}{9 \pi c}\right)^{2}} \\
-\frac{16 b}{9 \pi c}, \\
\quad \theta=\frac{1}{2} \arcsin \left(\frac{4 C \Delta}{a k_{c} V_{\mathrm{in}}}\right)+n \pi .
\end{gathered}
$$

As solution (7) is defined only if $|\Delta|<a k_{c} V_{\text {in }} /(4 C)$, it exists only within the following frequency range:

$$
2|\Delta|_{\operatorname{MAX}}=\frac{a k_{c} V_{\text {in }}}{2 C}
$$

centred around $\omega=\omega_{0}$. In order to investigate the stability of solution (7) in the interval of existence (8), we linearize the nonlinear system (6) around the solution (7) and calculate the eigenvalues of the corresponding Jacobian matrix. Defining $m=k_{c} V_{\text {in }} / 4$ and $\Delta_{n}=C \Delta / a$, we obtain the following eigenvalues:

$$
\begin{gathered}
\lambda_{a}=-2 m \sqrt{1-\left(\frac{\Delta_{n}}{m}\right)^{2}}, \\
\lambda_{b}=\lambda_{a}-\left(1-\frac{1}{a R}-\frac{4 b}{3 \pi} V\right) .
\end{gathered}
$$

Taking into account the start-up condition of the basic unforced $L C$ differential oscillator, that $k<0$, and hence, $m>0$, both of the eigenvalues are negative within the interval of existence of the solution. Thus, we conclude that (8) provides the frequency interval wherein the injectionlocked frequency divider is able to lock the input signal, that is, the so-called locking range. Expression (8) clearly shows that the locking range of direct-injection frequency dividers, differently from the locking range of frequency dividers with injection via tail device $[6,9]$, does not depend on the circuit losses, and thus, it can be widened without lowering the quality factor and the phase noise of the divider.

Numerical integration of system (6) confirms the validity of formulas (7)-(9), as shown in Figure 7, where the phase portrait of the system is represented for two set of parameters, the first of which corresponds to the second set of experimental measurements shown in Figures 8(a) and 8(b).

Finally, note that the presented formulas provide several interesting and useful design insights to circuit designers, which are usually interested in design variables such as oscillation amplitude, lock range, and stability of lock, which are here given in a simple explicit form in terms of circuit parameters. 


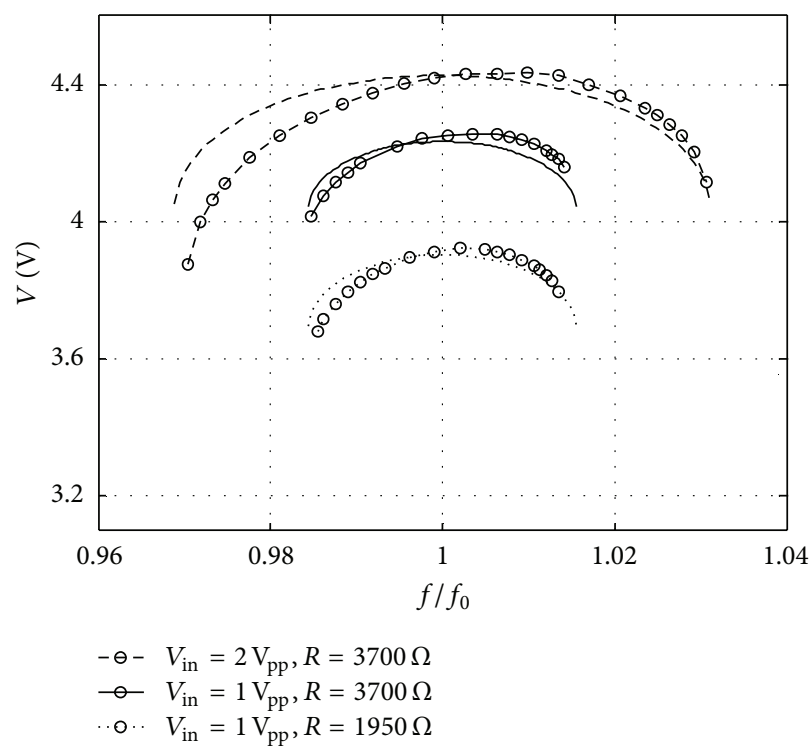

(a)

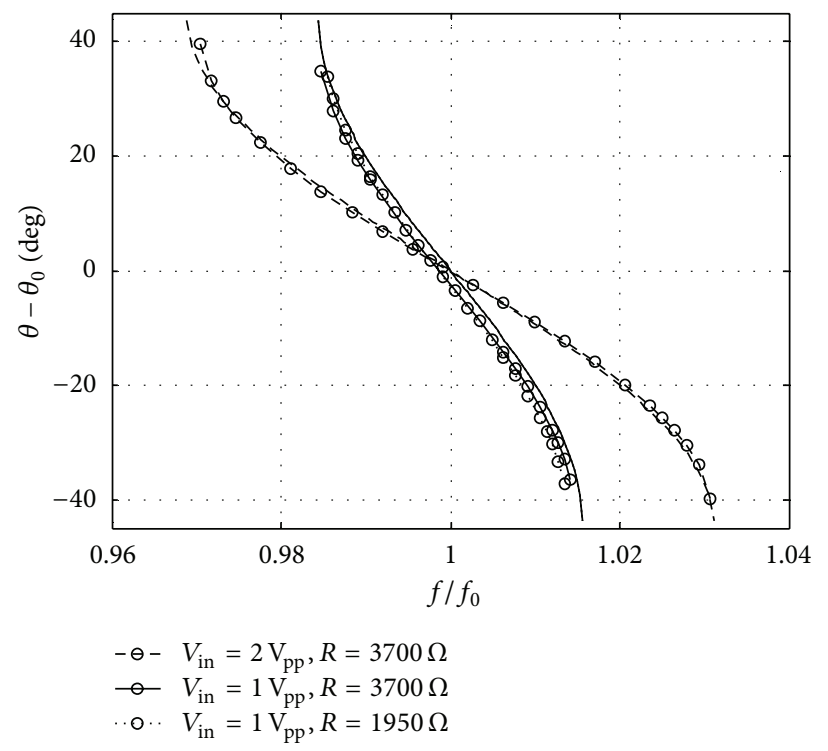

(b)

FIgURE 8: (a) Steady-state amplitude and (b) phase for the ILFD in Figure 1 with $V_{\mathrm{DD}}=5 \mathrm{~V}, V_{0}=1 \mathrm{~V}, L=4.759 \mu \mathrm{H}$, and $C=110 \mathrm{pF}$ $\left(f_{0}=6.956 \mathrm{MHz}\right.$ ). Results are obtained by measurements (lines with circles) using integrated circuits ST HCF4007UB and by (7) (lines without circles) using parameter values deduced by Figures 5 and 6 .

\section{Experimental Results}

In order to experimentally verify the circuit model and the presented formulas, a prototype of the circuit in Figure 1 was implemented using integrated circuits ST HCF4007UB (dual complementary pairs plus inverter) powered by a $V_{\mathrm{DD}}=5 \mathrm{~V}$ battery. The RLC tank was realized using an RF choke of BC series by Epcos, a ceramic disc capacitor of 140-500 N5 series by Xicon and a metal film fixed resistor of MF-RC series by Xicon.

The presented methodology for predicting the behavior of the injection-locked frequency divider provides the analytical expressions for the output amplitude $V$, the phase $\theta$, and the locking range $L R=2|\Delta|_{\mathrm{MAX}}$ as a function of the active and passive circuit parameters. In particular, the active parameters of the ILFD appear through the parameters of the nonlinearities, that is, $a_{\mathrm{nl}}, c_{\mathrm{nl}}, a_{\mathrm{in}}, b_{\mathrm{in}}, c_{\mathrm{in}}$, and $k$. Thus, we first determined the parameters of the nonlinearities using the auxiliary circuit in Figure 4 , obtaining $a_{\mathrm{nl}}=-1.94 \mathrm{~mA} / \mathrm{V}$ and $c_{\mathrm{nl}}=80 \mu \mathrm{A} / \mathrm{V}^{3}$ for the nonlinear characteristic of the locally active two-terminal sub-circuit, and $a_{\mathrm{in}}=0.13 \mathrm{~mA} / \mathrm{V}$, $b_{\text {in }}=40 \mu \mathrm{A} / \mathrm{V}^{2}, c_{\text {in }}=35 \mu \mathrm{A} / \mathrm{V}^{3}$, and $k=-0.6 \mathrm{~mA} / \mathrm{V}^{2}$ for the nonlinear characteristic of the injection circuit. The equivalent capacitance due to the combined effect of the tank capacitor, the active device parasitics, and the layout parasitics was determined by measuring the actual free-running oscillation frequency $f_{0}=\omega_{0} /(2 \pi)$, that is, the output divider frequency when $v_{\text {in }}=0$. The overall tank resistance was estimated by measurements at the tank resonant frequency.

Then, we compared the results obtained by formulas (7) with the measurements of the output amplitude and phase of the circuit in Figure 1. The results are shown in Figure 8, for different values of the amplitude of the input signal, $V_{\text {in }}$, the frequency of the input signal, $f_{\text {in }}=2 f$, and circuit losses, $R$. Figures $8(\mathrm{a})$ and $8(\mathrm{~b})$ show that formulas (7) proved an overall acceptable estimation. Moreover, it is interesting to observe that a quite good approximation to measurement results is obtained also by formula (8) for the locking ranges, which are represented in Figures 8(a) and 8(b), by the width of the frequency intervals, over which the analytical and numerical results are available. However, formulas are not able to predict a constant phase offset $\theta_{0}=5^{\circ}$, that is, due to the reactive behavior of nonlinearities, which were here assumed to be memoryless. Finally, it should be observed that the accuracy of amplitude formula is better for small injection values as it is derived assuming small injection values and neglecting output harmonics [21-26]. Thus, we can conclude that, even if small discrepancies are present between experimental and analytical results, formulas are able to capture the main characteristics of the injectionlocking phenomenon, providing a quite good approximation to experimental results.

Finally, it should be highlighted that even if our experiments were performed in $\mathrm{MHz}$ range as in $[8,16]$, similar results are expected at $\mathrm{GHz}$ range because it was shown in [5] that approximate models based on algebraic characteristics for the active parts of the divider can be effectively used at $\mathrm{GHz}$ frequencies.

\section{Conclusions}

We have experimentally verified the possibility of predicting accurately the behavior of injection-locked frequency dividers with direct injection through simple formulas that provide useful design insights to circuit designers. This is 
made possible by the dynamical model (6) of frequency dividers that describes their nonlinear dynamic to first-order, according to the well-known averaging method. This method is a powerful tool since it allows us to predict not only the oscillations in the synchronized states and their stability, which have been analyzed in this investigation, but also their transient behavior before reaching the steady states.

\section{References}

[1] R. Adler, "A study of locking phenomena in oscillators," Proceedings of the IRE, vol. 34, pp. 351-357, 1946.

[2] K. Kurokawa, "Injection locking of microwave solid-state oscillators," Proceedings of the IEEE, vol. 61, no. 10, pp. 1386-1410, 1973.

[3] K. Kurokawa, "Stability of injection-locked oscillators," Proceedings of the IEEE, vol. 60, pp. 907-908, 1972.

[4] T. Shibasaki, H. Tamura, K. Kanda, H. Yamaguchi, J. Ogawa, and T. Kuroda, "20-GHz quadrature injection-locked LC dividers with enhanced locking range," IEEE Journal of Solid-State Circuits, vol. 43, no. 3, pp. 610-618, 2008.

[5] H. R. Rategh and T. H. Lee, "Superharmonic injection-locked frequency dividers," IEEE Journal of Solid-State Circuits, vol. 34, no. 6, pp. 813-821, 1999.

[6] S. Verma, H. R. Rategh, and T. H. Lee, "A unified model for injection-locked frequency dividers," IEEE Journal of Solid-State Circuits, vol. 38, no. 6, pp. 1015-1027, 2003.

[7] F. Ramírez, M. E. De Cos, and A. Suárez, "Nonlinear analysis tools for the optimized design of harmonic-injection dividers," IEEE Transactions on Microwave Theory and Techniques, vol. 51, no. 6, pp. 1752-1762, 2003.

[8] S. Daneshgar, O. De Feo, and M. P. Kennedy, "Observations concerning the locking range in an LC injection-locked frequency divider-part I: qualitative analysis," IEEE Transactions on Circuits and Systems I, vol. 57, no. 1, pp. 179-188, 2010.

[9] A. Buonomo and A. Lo Schiavo, "Analytical approach to the study of injection-locked frequency dividers," IEEE Transactions on Circuits and Systems-I, vol. 60, no. 1, pp. 51-62, 2013.

[10] A. Buonomo and A. Lo Schiavo, "Nonlinear dynamics of divideby-two injection-locked frequency dividers in locked operation mode," International Journal of Circuit Theory and Applications, 2013.

[11] M. Tiebout, "A CMOS direct injection-locked oscillator topology as high-frequency low-power frequency divider," IEEE Journal of Solid-State Circuits, vol. 39, no. 7, pp. 1170-1174, 2004.

[12] C. Y. Wu and C. Y. Yu, "Design and analysis of a millimeter-wave direct injection-locked frequency divider with large frequency locking range," IEEE Transactions on Microwave Theory and Techniques, vol. 55, no. 8, pp. 1649-1657, 2007.

[13] S. Daneshgar and M. P. Kennedy, "Analysis of Forward Body Biasing (FBB) in a complementary differential LC injectionlocked frequency divider based on direct injection," in Proceedings of the 6th Conference on Ph.D. Research in Microelectronics and Electronics (PRIME '10), Berlin, Germany, July 2010.

[14] A. Buonomo and A. Lo Schiavo, "Finding the tuning curve of a CMOS-LC VCO," IEEE Transactions on Circuits and Systems II, vol. 55, no. 9, pp. 887-891, 2008.

[15] A. Buonomo and A. Lo Schiavo, "The effect of parameter mismatches on the output waveform of an LC-VCO," International Journal of Circuit Theory and Applications, vol. 38, no. 5, pp. 487501, 2010.
[16] S. Daneshgar, O. De Feo, and M. P. Kennedy, "Observations concerning the locking range in a complementary differential LC injection-locked frequency divider-part II: design methodology," IEEE Transactions on Circuits and Systems I, vol. 58, no. 4, pp. 765-776, 2011.

[17] A. Buonomo and A. Lo Schiavo, "On the theory of quadrature oscillations obtained through parallel LC VCOs," IEEE Transactions on Circuits and Systems I, vol. 57, no. 9, pp. 2509-2519, 2010.

[18] A. Buonomo and A. Lo Schiavo, "Modelling and analysis of differential VCOs," International Journal of Circuit Theory and Applications, vol. 32, no. 3, pp. 117-131, 2004.

[19] S. K. Magierowski and S. Zukotynski, "CMOS LC-oscillator phase-noise analysis using nonlinear models," IEEE Transactions on Circuits and Systems I, vol. 51, no. 4, pp. 664-677, 2004.

[20] A. Buonomo and A. Lo Schiavo, "Analyzing the dynamic behavior of RF oscillators," IEEE Transactions on Circuits and Systems I, vol. 49, no. 11, pp. 1525-1534, 2002.

[21] A. Buonomo, M. P. Kennedy, and A. Lo Schiavo, "On the synchronization condition for superharmonic coupled QVCOs," IEEE Transactions on Circuits and Systems I, vol. 58, no. 7, pp. 1637-1646, 2011.

[22] A. Buonomo and A. Lo Schiavo, "A deep investigation of the synchronization mechanisms in LC-CMOS frequencydividers," IEEE Transactions on Circuits and Systems -I. In press.

[23] P. Wambacq and W. Sansen, Distortion Analysis of Analog Integrated Circuits, Kluwer, Norwell, Mass, USA, 1998.

[24] A. Buonomo and A. Lo Schiavo, "Perturbation analysis of nonlinear distortion in analog integrated circuits," IEEE Transactions on Circuits and Systems I, vol. 52, no. 8, pp. 1620-1631, 2005.

[25] A. Buonomo and A. Lo Schiavo, "Nonlinear distortion analysis via perturbation method," International Journal of Circuit Theory and Applications, vol. 38, no. 5, pp. 515-526, 2010.

[26] A. Buonomo and A. Lo Schiavo, "A method for analysing the transient and the steady-state oscillations in third-order oscillators with shifting bias," International Journal of Circuit Theory and Applications, vol. 29, no. 5, pp. 469-486, 2001. 

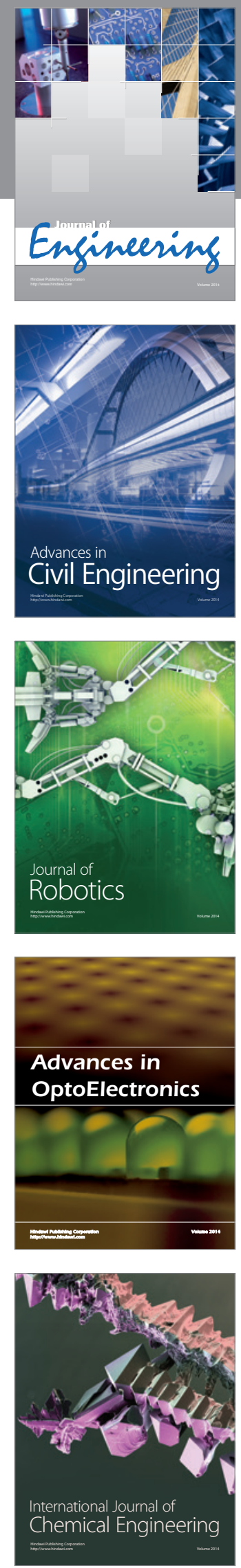

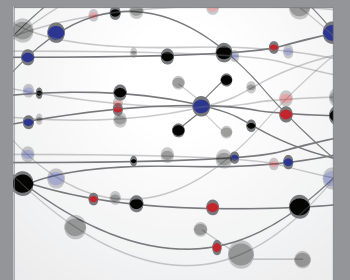

The Scientific World Journal
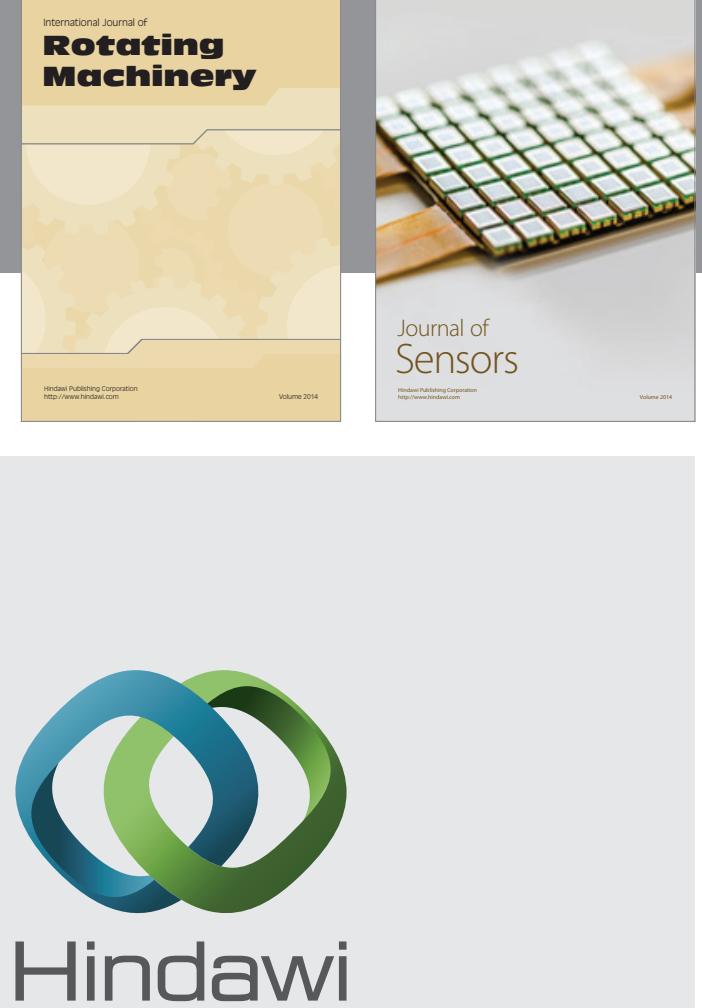

Submit your manuscripts at http://www.hindawi.com
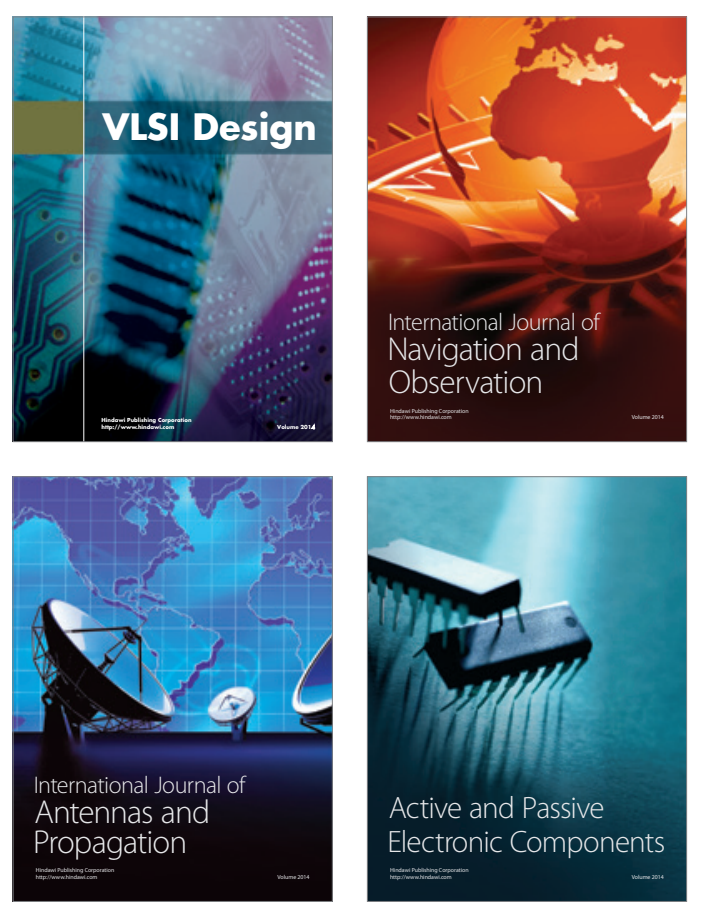
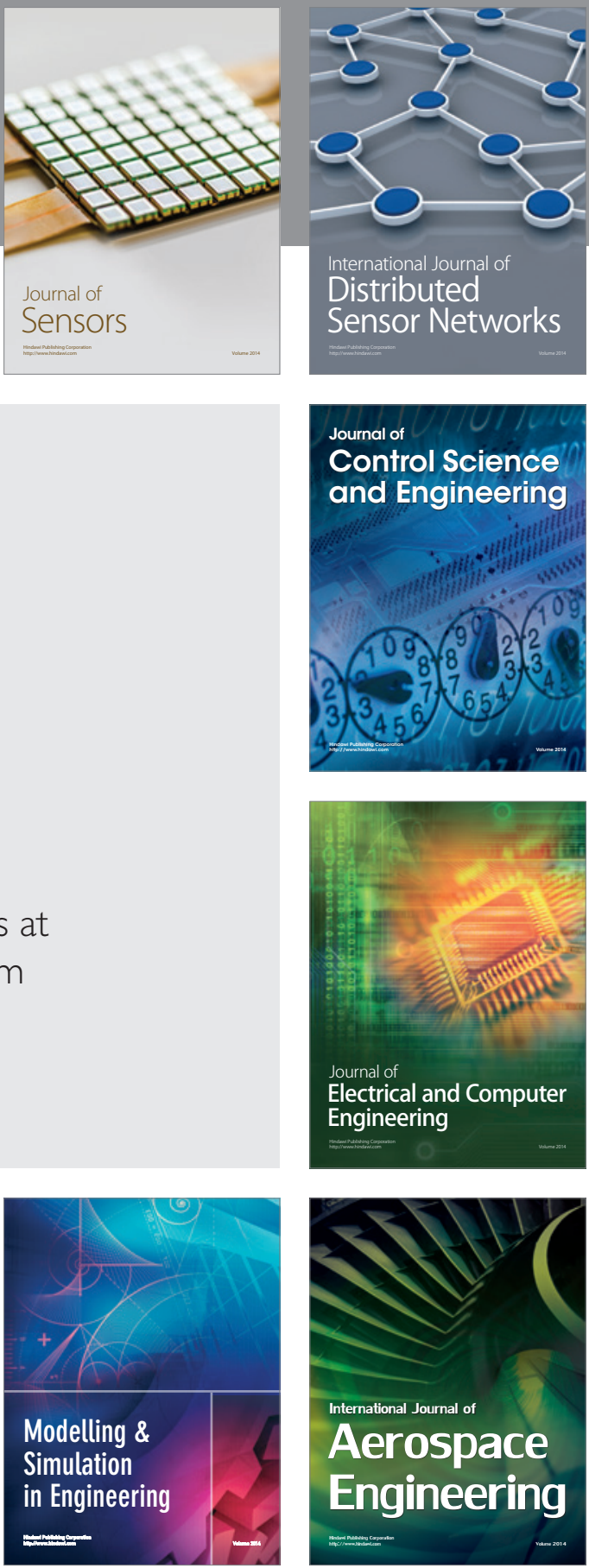

Journal of

Control Science

and Engineering
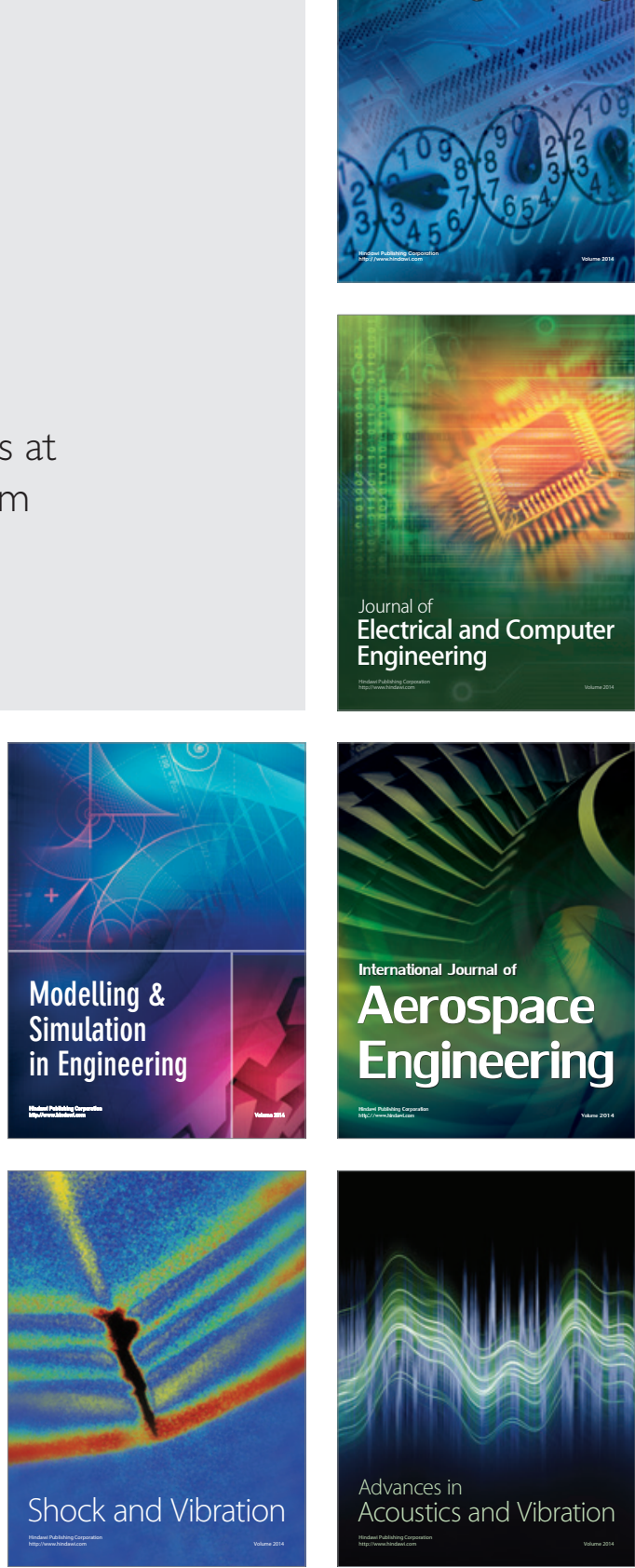